\title{
Akne - Zeichen des gestörten Gleichgewichts
}

\author{
Ernährung und ein alkalischer pH-Wert der Haut belasten den Stoffwechsel
}

Während das Potential von Kosmetika, Akne zu fördern in jüngerer Zeit als eher gering erkannt wurde, stellen kosmetische Hautpflegeprodukte für die Reinigung und Pflege eine sinnvolle Unterstützung der pharmakologischen Behandlung dar und sollten auf diese abgestimmt sein. Darüberhinaus gilt der zugrundeliegenden ernährungsbedingten Stoffwechselentgleisung, die sich in Akne ausdrücken kann, verstärktes Augenmerk.

Geeignete Kosmetika zur Reinigung und Pflege der Haut können bei Menschen mit Akneneigung zu einer Abnahme v. a. entzündlicher Läsionen beitragen, stellen Prof. Dr. Hans-Christian Korting, Klinikum der Ludwig-Maximilians-Universität München, und Mitarbeiter fest. Sie können freilich bei bestehender Akne eine pharmakologische Therapie nicht ersetzen, sondern diese sinnvoll ergänzen. Bestimmte Reinigungspräparate sind in der Lage, bei zu Akne neigender Haut die übermäßige Vermehrung von Propionibacterium acnes (P. acnes) durch Änderungen des Säuremilieus der Haut zu mindern und dadurch letztlich auch die Zahl der entzündlichen Läsionen zu reduzieren. So kommt es bei pH-Werten im Neutralbereich zu vermehrtem Wachstum der Aknebakterien. Da also das Säuremilieu eine entscheidende Rolle spielt, ist es günstig, bei zur Akne neigender Haut Hautreinigungs- und -pflegepräparate zu bevorzugen, die das Säuremilieu der Hautoberfläche möglichst wenig stören

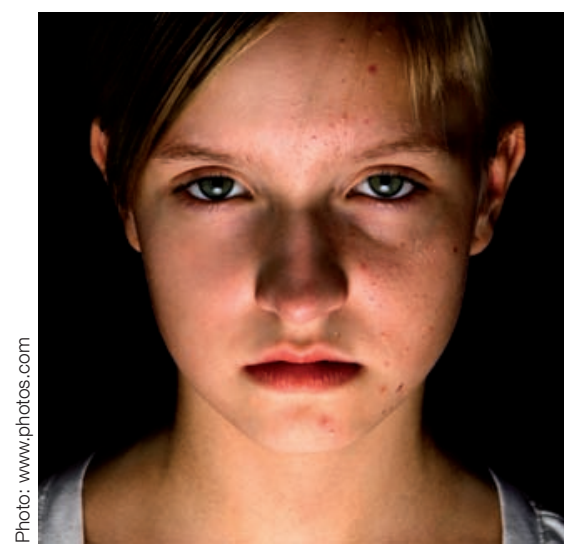

Abb. 1: Geeignete Kosmetika können eine pharmakologische Therapie nicht ersetzen, aber sinnvoll ergänzen. und die Hautintegrität nicht beeinträchtigen. Es konnte nachgewiesen werden, dass die zweimal tägliche Anwendung eines sauren Syndets mit einem pH-Wert von $5,5 \mathrm{zu}$ einer Verminderung inflammatorischer Läsionen bei Akneneigung führt, eine alkalische Seife jedoch nicht. Ein sauer eingestelltes Hautreinigungsmittel wirkt sich demnach, so Korting et al., anders als alkalische Seife bei Akneneigung günstig auf den Hautzustand auswirkt.

Zur Pflege von Haut mit Akneneigung können je nach Hautzustand unterschiedliche Formulierungstypen eingesetzt werden, etwa Hydrogele, Gelcremes oder Cremes. Dabei sollten im allgemeinen feuchtigkeitsspendende Produkte zum Einsatz kommen, die hydrophil und nicht fettend sein sollten. „Da die Barrierefunk-

\section{Die Haut gilt als Spiegel der Seele, die Akne ist aber vor allem ein Spiegel westlicher Fehlernährung.}

tion der Haut beeinträchtigt sein kann, können auch leichte, nichtkomedogene Emollienzien verwendet werden“, stellen die Autoren fest. Zudem können keratolytisch wirkende Inhaltsstoffe wie Alphahydroxysäuren, Salizylsäure, Lipohydroxysäure und Retinaldehyd eingesetzt werden, ferner Peeling-Verfahren und Masken. Wissenschaftlichen Untersuchungen zufolge wirken darüber hinaus Inhaltsstoffe, wie beispielsweise Nicotinamid, Milchsäure, Triethylacetat/Ethyllineolat und präbiotisch wirksame Pflanzenextrakte günstig auf verschiedene Mechanismen der Aknepathogenese ein und Hautpflegepräparate, die diese enthalten, können das Auftreten von Läsionen vermindern helfen.

Als einen der wesentlichsten Umweltfaktoren konnte man die Ernährung des westlichen Lebensstils identifizieren. Dementsprechend kann eine Umstellung der Ernährung bedeutende Verbesserungen des Hautzustands erzielen. Neuere Ernährungsstudien bestätigen die angenommene Bedeutung von Milchprodukten und Kohlenhydraten mit hohem Glykämischem Index (G.I.) als Auslöser der Akne, stellt Prof. Bodo Melnik von der Universität Os- nabrück fest und fügt hinzu, dass ein weiterer Risikofaktor westlichen Lebensstils der gesteigerte Konsum insulinotroper Molkeproteinkonzentrate im Fitnessumfeld ist, der oft in Kombination mit illegalem Androgenabusus sowie der Verwendung von synthetischem Wachstumshormon (GH), Insulin und insulinartigem Wachstumsfaktor-1 (IGF-1) einhergeht. Dabei wirken alle nutritiven Akneauslöser sowie auch das Rauchen synergistisch und potenzierend über den aus der Onkologie gut bekannten Wachstumsfaktor-Phosphoinositol-3-Kinase (PI3 K)/Akt-Signalweg.

Der Ernährung sollte daher bei der Beratung und Behandlung von Aknepatienten ein besonderer Stellenwert eingeräumt werden. Im Vordergrund, so Melnik, stehen die Reduktion des Konsums von Milch

und Milchprodukten, die Einschränkung hyperglykämischer Nahrungsmittel, der Verzicht auf Fast Food, die Verminderung des Konsums von Milchschokolade, Süßigkeiten, zuckerhaltigen Limonaden und tierischen Fetten mit hohem Gehalt an Arachidonsäure sowie die Abstinenz vom Rauchen. „Die Haut gilt als Spiegel der Seele, was für die stressinduzierte Akne besonders zutrifft", konstatiert Melnik: „Die Akne ist aber vor allem ein Spiegel westlicher Fehlernährung."

\section{LITERATUR}

H.C. Korting et al. Acne vulgaris. Rolle der Kosmetik, Hautarzt 2010; 61:126-131

B. Melnik Acne vulgaris. Rolle der Diät, Hautarzt $2010 \cdot 61: 115-125$

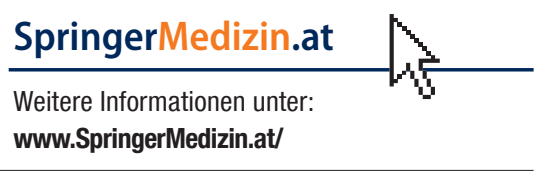

